\title{
Simetría del estado de los primeros molares permanentes en adolescentes mexicanos de 12 y 15 años de edad
}

\author{
Symmetry State of the First Permanent Molars of Mexican Adolescents 12 and 15 Years Old
}

\author{
Víctor Manuel Fernández-Hernández ${ }^{1}$, América Patricia Pontigo-Loyola ${ }^{2}$, \\ María de Lourdes Márquez-Corona ${ }^{3}$, Carlo Eduardo Medina-Solís ${ }^{4}$,Edith Lara-Carrillo ${ }^{5}$, \\ Mirna Minaya-Sánchez ${ }^{6}$, Juan Fernando Casanova-Rosado ${ }^{7}$, Alejandro José Casanova- \\ Rosado $^{8}$
}

\begin{abstract}
:
Objective: To identify the existence of symmetry in the state (healthy or caries experience) who keep the first permanent molars (FPMs) in Mexican adolescents. Material and Methods: A cross-sectional study was performed where 1,538 adolescents aged 12 and 15 years old from a community of Hidalgo, Mexico were included. Through a clinical examination, the status of FPMs as to whether was healthy or with sealant, in addition to its present caries experience (decayed tooth) and past (filled tooth or extracted tooth) was determined. To determine the symmetry in the state of left-right molars, statistical analysis was performed using tests of StuartMaxwell and McNemar in Stata 11. Results: Of the 1538 adolescents, 688 were 12 years and 85015 years of age. Girls accounted for 49.9\%. It was observed that $56.8 \%$ had four FPMs without caries experience and $4.9 \%$ had affected the four FPMs. The results of Stuart-Maxwell and McNemar tests showed presence of symmetry in the upper FPMs as P values were greater than 0.05 (no significant difference). However this situation was not observed for the lower FPMs, had no symmetry ( $\mathrm{p}<0.05$ ). Conclusions: This study suggests that the state of the upper FPMs have symmetry in terms of past and present caries experience. However, this situation in the lower FPMs was not observed.
\end{abstract}

\section{Keywords:}

${ }^{1}$ Alumno tesista del Área Académica de Odontología del Instituto de Ciencias de la Salud de la Universidad Autónoma del Estado de Hidalgo. Pachuca, México.fernandezhv@ hotmail.com

${ }^{2}$ Profesor-Investigador del Área Académica de Odontología del Instituto de Ciencias de la Salud de la Universidad Autónoma del Estado de Hidalgo. Pachuca, México. americap@uaeh.edu.mx

${ }^{3}$ Profesor-Investigador del Área Académica de Odontología del Instituto de Ciencias de la Salud de la Universidad Autónoma del Estado de Hidalgo.Pachuca, México.lulumaco_1@yahoo.com.mx

${ }^{4}$ Autor de Correspondencia Profesor-Investigador del Área Académica de Odontología del Instituto de Ciencias de la Salud de la Universidad Autónoma del Estado de Hidalgo. Pachuca, México. Profesor por asignatura del Centro de Investigación y Estudios Avanzados en Odontología "Dr. Keisaburo Miyata" de la Facultad de Odontología de la Universidad Autónoma del Estado de México. Toluca, México. Email: cemedinas@yahoo.com

${ }^{5}$ Profesor-Investigador del Centro de Investigación y Estudios Avanzados en Odontología "Dr. Keisaburo Miyata" de la Facultad de Odontología de la Universidad Autónoma del Estado de México. Toluca, México. edithlarac@yahoo.com

${ }^{6}$ Facultad de Odontología de la Universidad Autónoma de Campeche. Campeche, México. minardam@yahoo.com.mx Facultad de Odontología de la Universidad Autónoma de Campeche. Campeche, México. casanovajf@yahoo.com.mx

${ }^{8}$ Facultad de Odontología de la Universidad Autónoma de Campeche. Campeche, México. alejandro_casanova@yahoo.com.mx 


\begin{abstract}
Resumen:
Objetivo: Identificar la existencia de simetría en el estado (sanos o con experiencia de caries) que guardan los primeros molares permanentes (PMPs) en adolescentes mexicanos. Material y Métodos: Se realizó un estudio de diseño transversal donde se incluyeron 1,538 adolescentes de 12 y 15 años de edad de una comunidad de Hidalgo, México. A través de una exploración clínica se determinó el estado de los PMPs en cuanto a si estaba sano o con sellador, además de su experiencia de caries presente (diente cariado) y pasada (diente obturado o diente extraído). Para determinar la simetría en el estado de los molares derecho e izquierdo, el análisis estadístico se realizó utilizando las pruebas de simetría de Stuart-Maxwell y de McNemar en Stata 11. Resultados: De los 1538 adolescentes, 688 tenían 12 años de edad y 85015 años. Las niñas representaron 49.9\%. Se observó que $56.8 \%$ tuvieron sus cuatro PMPs sin experiencia de caries y $4.9 \%$ tuvo afectado los cuatro PMPs. Los resultados de las pruebas estadísticas mostraron la existencia de simetría en los PMPs superiores ya que los valores de p resultaron mayores a 0.05 (sin diferencia significativa). En cambio, esta situación no fue observada para los PMPs inferiores, no presentaban simetría $(\mathrm{p}<0.05)$. Al analizar por sexo y edad, en las mujeres y en los adolescentes de 12 años, no se observó simetría en los PMPs inferiores. Conclusiones: El presente trabajo sugiere que el estado de los PMPs superiores presentan simetría en cuanto a la experiencia de caries presente y pasada. En cambio, en los PMPs inferiores no se observó esa situación en todos los casos.
\end{abstract}

\title{
Palabras Clave:
}

salud bucal, primeros molares permanentes, escolares

\section{Introducción}

Los primeros molares permanentes (PMPs), son los primeros órganos dentales de la segunda dentición en erupcionar y acompañan a la dentición primaria en la boca de un niño, transformando con su presencia la dentición primaria en mixta (Escareño, 1990). El primer molar permanente inicia su organogénesis alrededor del cuarto mes de vida intrauterina, mientras que su calcificación se inicia en la semana 25 y finaliza a los 9 años de edad. Al nacimiento ya se puede constatar cierto grado de calcificación coronaria en la cúspide mesiovestibular, finalizando su calcificación alrededor de los 2.5 a 3 años de edad. La maduración del esmalte se produce aproximadamente 2 años después de su erupción y su formación radicular termina entre los 9 y 10 años de edad (Bharti-Yadav, Angadi, y Kummar-Yadav, 2015).

Los PMPs son los órganos dentales de mayor importancia de la dentición permanente, ya que cumplen con un papel fundamental en el desarrollo y fisiología del sistema estomatognático. De esta forma, se ha determinado que entre sus funciones más importantes están: 1) Representan alrededor del 50\% de la eficiencia masticatoria, 2) Determina el patrón de masticación durante toda la vida, 3) Sirven de guía de erupción para los demás dientes molares, 4) Son responsables del segundo levante fisiológico de la oclusión, y 5) Se les considera la llave de la oclusión de Angle (Isla \& Villacorta, 2014; Gómez-Capote, Hernández-Roca, León-Montano, CamachoSuárez, y Clausell-Ruiz, 2015). Por la edad en la que erupcionan y la circunstancia en que aparece en la boca sin haber exfoliado ningún elemento primario, los padres, muchas veces, desconocen que este es un diente permanente. Esto hace que el PMP esté sometido a factores de riesgo y sea susceptible a caries dental y el avance de la misma, con la consecuente destrucción y pérdida temprana. Lamentablemente, este primer exponente de la dentición permanente, tiene muy poco tiempo de vida sana en la boca de un niño (Gómez-Capote, Hernández-
Roca, León-Montano, Camacho-Suárez, y Clausell-Ruiz, 2015).

Estudiar los primeros molares es un factor predictivo para identificar el estado de salud dental en escolares. Al evaluar estos órganos dentarios se puede obtener información actualizada del estado de salud de la cavidad bucal en niños de once años, porque estos dientes han tenido un tiempo de exposición entre cuatro y cinco años a los factores cariogénicos (Oropeza-Oropeza, Molina-Frechero, Castañeda-Castaneira, Zaragoza-Rosado, y Cruz-Leyva, 2012). Epidemiológicamente, la presencia de simetría en el estado de salud/enfermedad de los primeros molares podría ayudar a disminuir el tiempo que se invierte en la recolección de información clínica en estudios sobre caries dental en grandes poblaciones. Por otro lado, se podría construir modelos de riesgo para caries dental, ya que se sabe que la experiencia previa de caries es un excelente indicador para caries futura.

Diversos estudios se han realizado sobre el estado de salud que guardan los PMP en diversas poblaciones (Pérez, Gutiérrez, Soto, Vallejos, y Casanova, 2002; Casanova-Rosado, MedinaSolis, Casanova-Rosado, y Vallejos-Sánchez, 2005; Xue, Lin, Jie, y Qing, 2015; Alwayli, Alshiha, Alfraih, Hattan, Alamri, y Aldossary, 2017), sin embargo no se han dirigido a investigar la existencia de si existe simetría en él. El objetivo del estudio fue identificar la existencia de simetría en el estado (sanos o con experiencia de caries) que guardan los primeros molares permanentes (PMPs) en adolescentes mexicanos.

\section{Material y Métodos}

\section{Diseño, población y muestra del estudio}

Se realizó un estudio transversal en escolares de 12 y 15 años de edad de primarias y secundarias registradas en Tula Centro, San Marcos y El Llano. Tula de Allende es uno de los 84 municipios del Estado de Hidalgo, México. La metodología ha sido publicada previamente (Pontigo-Loyola, Medina-Solís, Márquez-Corona, Vallejos-Sánchez, Minaya-Sánchez, 
Escoffié-Ramirez, y Maupomé, 2012). La población incluyó inicialmente a 1,768 escolares. Siete escuelas no autorizaron el permiso para realizar la inspección bucal de los escolares, quedando excluidos 139 escolares (7.9\%). Fueron examinados 1,629 escolares inscritos en las 25 escuelas que participaron en este estudio. Se excluyeron 91 escolares, por diversas razones. La tasa de no respuesta para el examen bucal clínico fue del $5.6 \%$. De esta forma, la muestra final quedó constituida por 1,538 adolescentes.

\section{Variables y recolección de datos}

La variable dependiente del presente análisis fue el estado de cada primer molar permanente. El cual fue calculado a partir del índice CPOD (índice de dientes cariados, perdidos y obturados), el cual mide la experiencia de caries presente (componente Dientes Cariados) y pasada (componentes Dientes Perdidos y Obturados) en la dentición permanente. Además también se recogió información sobre la presencia de selladores de fosetas y fisuras. Primero, se realizó una prueba piloto con la finalidad de estandarizar los criterios, así como para verificar la duración de los procedimientos llevados a cabo en los exámenes clínicos de los escolares. En la inspección clínica-dental se empleó sonda y espejo bucal del número cinco bajo luz de día. Los escolares fueron examinados dentro de las instalaciones de cada institución educativa, evitando en lo posible interferir con las actividades educativas propias de los escolares incluidos en este estudio. Con esta inspección se determinó la experiencia de caries (World Health Organization, 1997). Los exámenes bucales fueron realizados por dos examinadores capacitados y estandarizados en los criterios epidemiológicos utilizados en las encuestas de salud bucal (Kappa inter e intra examinador $>0.80)$.

\section{Análisis estadístico}

El análisis estadístico consistió en la determinación de frecuencias y porcentajes de las variables cualitativas y de promedios y desviación estándar de las variables cuantitativas. Para determinar la simetría en el estado de los molares derecho e izquierdo, el análisis estadístico se realizó utilizando las pruebas de Stuart-Maxwell y de McNemar en Stata 11.

\section{Consideraciones éticas}

La realización de este estudio cumplió con las especificaciones de protección a los participantes en estudios y se adhirió a las reglamentaciones éticas en vigor en la Universidad Nacional Autónoma de México y la Universidad Autónoma del Estado de Hidalgo.

\section{Resultados}

De los 1538 adolescentes, 688 tenían 12 años de edad y 85015 años. Las niñas representaron $49.9 \%$. Se observó que $56.8 \%$ tuvieron sus cuatro PMPs sin experiencia de caries y $4.9 \%$ tuvo afectado los cuatro PMPs. El cuadro I muestra los resultados de la prueba estadística de Stuart-Maxwell para el total de la muestra, los primeros molares permanentes superiores no mostraron ser estadísticamente diferentes $(\mathrm{p}=0.6507)$ en el estado. Por otro lado, los primeros molares permanentes inferiores no presentaron simetría en su estado $(\mathrm{p}<0.05)$, su distribución mostró diferencias estadísticamente significativas.
Los resultados de las pruebas de simetría y McNemar mostraron la existencia de simetría en los PMPs superiores ya que los valores de $\mathrm{p}$ resultaron mayores a 0.05 (sin diferencia significativa). En cambio esta situación no fue observada para los PMPs inferiores, no presentaban simetría $(\mathrm{p}<0.05)$.

\begin{tabular}{|c|c|c|c|c|c|c|}
\hline & \multicolumn{6}{|c|}{ PMP superiores izquierdos } \\
\hline & & sano/sellador & cariado & obturado & perdido & total \\
\hline PMP & sano/sellador & 1246 & 72 & 19 & 5 & 1342 \\
\hline superiores & cariado & 76 & 51 & 7 & 1 & 135 \\
\hline \multirow[t]{6}{*}{ derechos } & obturado & 11 & 10 & 31 & 0 & 52 \\
\hline & perdido & 0 & 3 & 0 & 3 & 6 \\
\hline & total & 1333 & 136 & 57 & 9 & 1535 \\
\hline & & & & & & $p=0.6507$ \\
\hline & \multicolumn{6}{|c|}{ PMP inferiores izquierdos } \\
\hline & & sano/sellador & cariado & obturado & perdido & total \\
\hline PMP & sano/sellador & 959 & 145 & 18 & 6 & 1128 \\
\hline inferiores & cariado & 86 & 170 & 17 & 4 & 277 \\
\hline \multirow[t]{4}{*}{ derechos } & obturado & 29 & 16 & 65 & 1 & 111 \\
\hline & perdido & 5 & 7 & 1 & 7 & 20 \\
\hline & total & 1079 & 338 & 101 & 18 & 1536 \\
\hline & & & & & & $p=0.0035$ \\
\hline
\end{tabular}

En el cuadro II se muestran los resultados con relación al sexo y en el cuadro III los relacionados a con la edad. En cuanto al sexo, entre los hombres, los PMPs superiores y los PMPs inferiores mostraron ser simétricos en su estado. Por el contrario, entre las mujeres, sólo se observó simetría en los PMPs superiores. Referente a la edad, en los adolescentes de 12 años, el estado de los primeros molares superiores mostró ser simétrico, en cambio, el estado de los primeros molares inferiores no resultó simétrico. A los 15 años, tanto los primeros molares superiores e inferiores fueron simétricos en el estado. Cuadro ll. Distribución del estado de los primeros molares permanentes en la muestra por sexo. Hombres PMP superiores izquierdos

\begin{tabular}{lllllll}
\hline & & sano/sellador & cariado & obturado & perdido & total \\
\hline PMP & sano/sellador & 626 & 37 & 9 & 2 & 674 \\
\cline { 2 - 7 } superiores & cariado & 37 & 26 & 5 & 1 & 69 \\
\cline { 2 - 7 } derechos & obturado & 4 & 3 & 14 & 0 & 21 \\
& perdido & 0 & 2 & 0 & 1 & 3 \\
& total & 667 & 68 & 28 & 4 & 767 \\
& & & & & & $\mathrm{p}=0.4579$
\end{tabular}

\begin{tabular}{|c|c|c|c|c|c|c|}
\hline & \multicolumn{6}{|c|}{ PMP inferiores izquierdos } \\
\hline & & sano/sellador & cariado & obturado & perdido & total \\
\hline \multirow{5}{*}{$\begin{array}{l}\text { PMP } \\
\text { inferiores } \\
\text { derechos }\end{array}$} & sano/sellador & 491 & 73 & 6 & 2 & 572 \\
\hline & cariado & 43 & 91 & 9 & 1 & 144 \\
\hline & obturado & 14 & 6 & 27 & 0 & 47 \\
\hline & perdido & 2 & 1 & 0 & 3 & 6 \\
\hline & total & 550 & 171 & 42 & 6 & 769 \\
\hline
\end{tabular}

\begin{tabular}{|c|c|c|c|c|c|c|}
\hline \multirow[t]{2}{*}{ Mujeres } & & \multicolumn{4}{|c|}{ PMP superiores izquierdos } & \multirow[b]{2}{*}{ total } \\
\hline & & sano/sellador & cariado & obturado & perdido & \\
\hline PMP & sano/sellador & 620 & 35 & 10 & 3 & 668 \\
\hline superiores & cariado & 39 & 25 & 2 & 0 & 66 \\
\hline \multirow[t]{6}{*}{ derechos } & obturado & 7 & 7 & 17 & 0 & 31 \\
\hline & perdido & 0 & 1 & 0 & 2 & 3 \\
\hline & total & 666 & 68 & 29 & 5 & 768 \\
\hline & & & & & & $p=0.7544$ \\
\hline & \multicolumn{6}{|c|}{ PMP inferiores izquierdos } \\
\hline & & sano/sellador & cariado & obturado & perdido & total \\
\hline PMP & sano/sellador & 468 & 72 & 12 & 4 & 556 \\
\hline inferiores & cariado & 43 & 79 & 8 & 3 & 133 \\
\hline \multirow[t]{4}{*}{ derechos } & obturado & 15 & 10 & 38 & 1 & 64 \\
\hline & perdido & 3 & 6 & 1 & 4 & 14 \\
\hline & total & 529 & 167 & 59 & 12 & 767 \\
\hline & & & & & & $p=0.0430$ \\
\hline
\end{tabular}


Cuadro III. Distribución del estado de los primeros molares permanentes en la muestra por edad.

\begin{tabular}{|c|c|c|c|c|c|c|}
\hline \multirow[t]{2}{*}{12 años } & \multicolumn{6}{|c|}{ PMP superiores izquierdos } \\
\hline & & sano/sellador & cariado & obturado & perdido & total \\
\hline \multirow{5}{*}{$\begin{array}{l}\text { PMP } \\
\text { superiores } \\
\text { derechos }\end{array}$} & sano/sellador & 585 & 26 & 8 & 1 & 620 \\
\hline & cariado & 24 & 17 & 4 & 0 & 45 \\
\hline & obturado & 3 & 3 & 13 & 0 & 19 \\
\hline & perdido & 0 & 0 & 0 & 2 & 2 \\
\hline & total & 612 & 46 & 25 & 3 & $\begin{array}{l}686 \\
p=0.3611\end{array}$ \\
\hline
\end{tabular}

PMP inferiores izquierdos

\begin{tabular}{lllllll}
\hline & & sano/sellador & cariado & obturado & perdido & total \\
\hline \multirow{2}{*}{$\begin{array}{l}\text { PMP } \\
\text { inferiores }\end{array}$ derechos } & sanolsellador & 447 & 73 & 5 & 0 & 525 \\
\cline { 2 - 7 } & cariado & 37 & 74 & 5 & 1 & 117 \\
\cline { 2 - 7 } & obturado & 7 & 7 & 25 & 0 & 39 \\
\cline { 2 - 7 } & perdido & 2 & 2 & 0 & 1 & 5 \\
\hline & total & 493 & 156 & 35 & 2 & 686 \\
\hline
\end{tabular}

\begin{tabular}{llllll} 
total & 493 & 156 & 35 & 2 & 686 \\
\hline 15 años & \multicolumn{4}{c}{} & \\
\hline
\end{tabular}

\begin{tabular}{|c|c|c|c|c|c|c|}
\hline \multirow[t]{2}{*}{15 años } & \multicolumn{6}{|c|}{ PMP superiores izquierdos } \\
\hline & & sano/sellador & cariado & obturado & perdido & total \\
\hline PMP & sano/sellador & 661 & 46 & 11 & 4 & 722 \\
\hline superiores & cariado & 52 & 34 & 3 & 1 & 90 \\
\hline \multirow[t]{6}{*}{ derechos } & obturado & 8 & 7 & 18 & 0 & 33 \\
\hline & perdido & 0 & 3 & 0 & 1 & 4 \\
\hline & total & 721 & 90 & 32 & 6 & 849 \\
\hline & & & & & & $p=0.9104$ \\
\hline & \multicolumn{6}{|c|}{ PMP inferiores izquierdos } \\
\hline & & sano/sellador & cariado & obturado & perdido & total \\
\hline PMP & sano/sellador & 512 & 72 & 13 & 6 & 603 \\
\hline inferiores & cariado & 49 & 96 & 12 & 3 & 160 \\
\hline \multirow[t]{4}{*}{ derechos } & obturado & 22 & 9 & 40 & 1 & 72 \\
\hline & perdido & 3 & 5 & 1 & 6 & 15 \\
\hline & total & 586 & 182 & 66 & 16 & 850 \\
\hline & & & & & & $p=0.3079$ \\
\hline
\end{tabular}

\section{Discusión}

Este estudio se propuso determinar la existencia de simetría en cuanto a la salud/enfermad (estado) que presenta el primer molar permanente. El primer molar permanente es una de las estructuras dentarias más importantes para el desarrollo de una oclusión adecuada, pues al ocupar un gran espacio en el sector posterior, su presencia es básica para el desarrollo y equilibrio de la oclusión. La caries dental y la enfermedad periodontal son las principales enfermedades bucales que presenta la población en general, de hecho, se reconocen como problemas de salud pública bucal.

La simetría tiene 2 muy importantes conceptos diferentes: 1) El de las justas proporciones, que se refiere a cualquier cosa bien equilibrada, bien proporcionada; y 2) el de la simetría bilateral o simetría de rotación, que hace referencia a la simetría de rotación, de izquierda y derecha, este segundo concepto es el de importancia para la realización de esta investigación. Se sabe que ciertas afecciones dentales tienden a presentarse simétricamente a través de los lados izquierdo y derecho de la boca, como la enfermedad periodontal y la pérdida de dientes. El presente trabajo sugiere que el estado de los PMPs superiores presentan simetría en cuanto a la experiencia de caries (medidos a través del índice CPOD), sin caries o con selladores. En cambio, en los PMPs inferiores no se observó esa situación. En general, al igual que Minaya-Sánchez, Vallejos-Sánchez, Casanova-Rosado, Casanova-Rosado, Medina-Solís, Maupomé, Márquez-Corona, e islas-Granillo, (2010); Wyne, (2004); Sansores-Ambrosio, Casanova-Rosado, VallejosSánchez, y Medina-Solís, (2003); Smith, Gran, y Cole, (2005);
Perzigian, (2005), que observaron simetría bilateral en otros eventos relacionados con la salud bucal, hemos encontrado que también el estado de los primeros molares permanentes sigue un patrón más o menos simétrico, con ciertas diferencias a los 12 años y en las mujeres.

Los resultados del presente estudio pueden ser útiles en estudios epidemiológicos sobre salud bucal, la presencia de simetría en el estado de salud/enfermedad de los primeros molares podría ayudar a disminuir el tiempo que se invierte en la recolección de información clínica en estudios sobre caries dental en grandes poblaciones. Con la información se podría construir modelos de riesgo para caries dental utilizando los primeros molares, ya que se sabe que la experiencia previa de caries es un excelente indicador para caries futura.

\section{Conclusiones}

Este estudio, de acuerdo al análisis realizado y dando respuesta a nuestra pregunta de investigación, podemos llegar a la conclusión de que, en general si existe una simetría en el estado de los Primeros Molares Permanentes. En las mujeres, no se observó en los PMPs inferiores. Lo mismo sucedió en los adolescentes de 12 años.

\section{Referencias}

1. Alwayli, H.M., Alshiha, S.A., Alfraih, Y.K., Hattan, M.A., Alamri, A.A., y Aldossary, M.S. (2017). A survey of fissure sealants and dental caries prevalence in the first permanent molars among primary school girls in Riyadh, Saudi Arabia. European Journal of Dentistry, 11, 455-460.

2. Bharti-Yadav, A., Angadi, P.V., y Kummar-Yadav, S. (2015). Sex assessment efficacy of permanent maxillary first molar cusp dimensions in Indians. Contemporary Clinical Dentistry, 6, 489495.

3. Casanova-Rosado, A.J., Medina-Solis, C.E., Casanova-Rosado, J.F., y Vallejos-Sánchez, A.A. (2005). Factores asociados a la pérdida del primer molar permanente en escolares de Campeche, México. Acta Odontológica Venezolana, 43, 268-275.

4. Escareño, C. (1990). Manual de Actividades Clínicas de Odontopediatría. México DF: Universidad Autónoma Metropolitana.

5. Gómez-Capote, I., Hernández-Roca, C.V., León-Montano, V., Camacho-Suárez, A.M. y Clausell-Ruiz, M. (2015). Caries dental en los primeros molares permanentes en escolares. Revista Médica Electrónica, 37, 207-217.

6. Isla, C., y Villacorta, C. (2014). Diagnóstico y evolución de primeros molares permanentes en niños en edad escolar. Acta Odontológica Venezolana, 52, disponible en: http://www.actaodontologica.com/ediciones/2014/3/art15.asp

7. Minaya-Sánchez, M., Vallejos-Sánchez, A.A., CasanovaRosado, A.J., Casanova-Rosado, J.F., Medina-Solís, C.E., Maupomé, G., Márquez-Corona, M.L., e islas-Granillo, H. (2010). Confirmation of symmetrical distributions of clinical attachment loss and tooth loss in a homogeneous Mexican adult male population. Journal of Dental Sciences, 5, 126-130.

8. Oropeza-Oropeza, A., Molina-Frechero, N., CastañedaCastaneira, E., Zaragoza-Rosado, Y., y Cruz-Leyva, D. (2012) Caries dental en primeros molares permanentes de escolares de la delegación Tláhuac. Revista ADM, 69, 63-68.

9. Pérez, S.A., Gutiérrez, M.P., Soto, L., Vallejos, A., y Casanova, J. (2002). Caries dental en primeros molares permanentes y factores socioeconómicos en escolares de Campeche, México. Revista Cubana Estomatología, 39, 265-281. 
10. Perzigian, A.J. (2005). Fluctuating dental asymemetry: Variation among skeletal populations. American Journal of Physical Anthropology, 47, 81-88.

11. Pontigo-Loyola, A.P., Medina-Solís, C.E., Márquez-Corona, M.L., Vallejos-Sánchez, A.A., Minaya-Sánchez, M., EscoffiéRamirez, M., y Maupomé G. (2012). Influencia de variables predisponentes, facilitadoras y de necesidades sobre la utilización de servicios de salud bucal en adolescentes mexicanos en un medio semi-rural. Gaceta Médica de México, $148,218-226$

12. Sansores-Ambrosio, F.E., Casanova-Rosado, J.F., VallejosSánchez, A.A., y Medina-Solís, C.E. (2003). Asimetría facial en escolares con clase I de Angle utilizando el panorograma de simetría de Simoes. Boletin Médico del Hospital Infantil de México; 60, 617-624.

13. Smith B.H., Garn S.M., y Cole P.E. (2005). Problems of sampling and inference in the study of fluctuating dental asymmetry. American Journal of Physical Anthropology, 58, 281-289.

14. World Health Organization. Oral Health Survey - Basics Methods. 4th ed. Geneva: World Health Organization, 1997.

15. Wyne, A.H. (2004). The bilateral occurrence dental caries among 12-13 and 15-19 years old school children. Journal of Contemporary Dental Practice, 5, 42-52.

16. Xue, Y., Lin, W., Jie, L., y Qing, D. (2015). Caries status of the first permanent molar among 7 - to 9-year-old children in Tangshan city and their correlation. Hua Xi Kou Qiang Yi Xue Za Zhi, 33, 54-7. 